\title{
Analysis of the Occurrence of Major Hemorrhagic Incidents in Patients with Low-Dose Aspirin Usage in Korean Popula- tions - Does Low-Dose Aspirin Really Increase the Risk of Bleeding?
}

\author{
Tae Gon Kim, $\mathrm{MD}^{1}$, Sang Heum Kim, $\mathrm{MD}^{2}$ \\ ${ }^{1}$ Department of Neurosurgery, CHA Bundang Medical Center, CHA University School of Medicine, Seongnam, Korea \\ ${ }^{2}$ Department of Neuroradiology, CHA Bundang Medical Center, CHA University School of Medicine, Seongnam, Korea
}

Aspirin, which has traditionally been used for analgesics and anti-inflammatory drugs, is known to have primary and secondary preventive effects as an antiplatelet agent against cerebrovascular diseases such as myocardial infarction, stroke, and transient cerebral ischemia. ${ }^{1,2}$ Low-dose aspirin, however, is known to increase the risk of gastrointestinal and intracranial hemorrhage, but there have been few studies on Korean populations, especially using large-scale data. We wanted to confirm the risk of lowdose aspirin bleeding in real Korean populations. Therefore, we have recently assessed the risk of hemorrhage (extracranial bleeding, such as gastrointestinal hemorrhage, and intracranial bleeding, such as spontaneous intracerebral hemorrhage) in patients who take low doses of aspirin using large-scale data.

In order to assess the bleeding risk of taking aspirin among Korean patients, we analyzed the National Inpatient Samples 2016 from the Healthcare Bigdata Hub of Health Insurance Review and Assessment Service (HIRA-NIS 2016), which best reflects the real world. HIRA-NIS 2016 data was extracted from a patient unit stratification system based on gender and age (5-year units), including details about care and prescriptions for all patients using hospitalization services throughout Korea for 1 year (2016). The extraction rate for inpatients was about $13 \%$, or about 1 million people. The subjects of this study were adults over 30 years old, and the data of 731,057 people (415,685 women and 315,372 men) were analyzed.

The dependent variable was bleeding, including intracranial or gastrointestinal hemorrhage, and only patients who were hospitalized with a diagnosis of bleeding were included with hemorrhages classified as present or absent. Patients hospitalized with hemorrhage before March 31, 2016 were excluded, since if the prescription period for aspirin was set at around 3 months, it would be unclear if aspirin use was the definitive cause of the hemorrhage. In addition, patients who had hemorrhages prior to 75 days (including an adaptation period of about 15 days and a treatment period of 60 days) after beginning an aspirin prescription were excluded. ${ }^{3}$ The KCD code used to diagnose bleeding

\section{Correspondence to: Tae Gon Kim, MD \\ Department of Neurosurgery, CHA Bundang Medical Center, CHA Univer- sity School of Medicine, 59 Yatap-ro, Bundang-gu, Seongnam 13496, Korea Tel: +82-31-780-5260 \\ Fax: +82-31-780-5269 \\ E-mail: tgkim@chamc.co.kr}

Received: December 31, 2019

Revised: January 24, 2020

Accepted: February 7, 2020

Copyright $\odot 2020$ Korean Society of Interventional Neuroradiology This is an Open Access article distributed under the terms of the Creative Commons Attribution Non-Commercial License (http://creativecommons.org/licenses/by-nc/3.0) which permits unrestricted non-commercial use, distribution, and reproduction in any medium, provided the original work is properly cited.

PISSN 2093-9043 eISSN 2233-6273 
was based on the disease name file published on July 27, 2017. Low-dose aspirin (less than $100 \mathrm{mg}$ ) was used as an independent variable, and the drug code was based on the list of drugs published on March 1, 2019. Confounding variables included gender, age, diabetes, hypertension, cerebrovascular disease (including myocardial infarction and cerebral infarction), usage of different types of antiplatelets, anticoagulants, nonselective nonsteroidal anti-inflammatory drugs (NSAIDs), aspirin as analgesic agents (more than $500 \mathrm{mg}$ ), systemic corticosteroids, selective serotonin reuptake inhibitors (SSRI), proton pump inhibitors (PPI), and HMG-CoA reductase inhibitors (statins). The age range was over 30 years old, and in case of confounding variables other than sex and age, the causal relationship was to be clarified by including only cases in which diagnosis or prescription was made before bleeding occurred. Age was classified in 1-year intervals, and all remaining confounding variables were classified as either present or absent or as either used or unused. For each of the confounding variables, chi-squared tests were performed to confirm the use of low-dose aspirin and its relevance to bleeding, and the relevance was verified via multiple logistic regression. All statistics were done using $R$ software version 3.6.1 (R Project for Statistical Computing, Vienna, Austria).

The chi-squared test results showed that the use of lowdose aspirin increases the risk of bleeding that required hospitalization (odds ratio [OR], 1.10; 95\% confidence interval [Cl], 1.04-1.16; $P=0.0004)$; but in multiple logistic regression analysis the adjusted $\mathrm{OR}$ was $0.81(95 \% \mathrm{Cl}, 0.76-0.87 ; \mathrm{P}=0.0000)$. In multiple logistic regression analysis, there were negative correlations between bleeding and the variables of aspirin use $(\mathrm{OR}, 0.81 ; 95 \% \mathrm{Cl}, 0.76-0.87 ; \mathrm{P}=0.0000)$, gender $(\mathrm{OR}, 0.77$; $95 \% \mathrm{Cl}, 0.73-0.80 ; \mathrm{P}=0.0000)$, diabetes $(\mathrm{OR}, 0.79 ; 95 \% \mathrm{Cl}$, $0.75-0.83 ; \mathrm{P}=0.0000)$, cerebrovascular disease $(\mathrm{OR}, 0.74 ; 95 \%$ $\mathrm{Cl}, 0.70-0.79 ; \mathrm{P}=0.0000)$, and NSAIDs (OR, 0.00; 95\% Cl, 0.00$0.00 ; P=0.0000$ ), since the $O R$ was less than 1. Conversely, variables such as age (OR, 1.01; $95 \% \mathrm{Cl}, 1.00-1.01 ; \mathrm{P}=0.0000)$, hypertension (OR, 1.21; 95\% Cl, 1.15-1.28; $\mathrm{P}=0.0000)$, other antiplatelets $(\mathrm{OR}, 1.17 ; 95 \% \mathrm{Cl}, 1.08-1.26 ; \mathrm{P}=0.0000)$, aspirin as analgesics (OR, 8,084.48; 95\% Cl, 6,101.54-10,711.85; $P=0.0000)$, steroids (OR, 1.67; 95\% Cl, 1.57-1.78; $\mathrm{P}=0.0000)$, SSRI (OR, 1.41; 95\% Cl, 1.29-1.54; $P=0.0000)$, PPI (OR, 1.71; 95\% Cl, 1.62-1.80; $\mathrm{P}=0.0000)$, and statins (OR, 1.30; 95\% Cl, 1.22-1.38; $\mathrm{P}=0.0000)$ had an OR larger than 1 . Therefore, it can be seen that there are positive correlations between these variables and bleeding.

There are many reports which have reported increased bleeding due to the use of aspirin. In 2009, meta-analysis data by the Antithrombotic Trialists' Collaboration reported higher annual incidences in patients treated with aspirin (0.10\% per year vs. $0.07 \%$ per year, $\mathrm{P}<0.0001)$. ${ }^{1}$ Similar results were also reported in a meta-analysis in 2016. In that report, the use of low-dose aspirin (less than $100 \mathrm{mg}$ per day) for the primary prevention of cardiovascular disease increased the risk of major gastrointestinal hemorrhage by $58 \%$ and the risk of hemorrhagic stroke by $27 \% .{ }^{2}$ In another meta-analysis in 2016, the relative risk of gastrointestinal hemorrhage due to low-dose aspirin was 1.4 overall, 2.3 and 1.8 for upper and lower gastrointestinal hemorrhage, respectively, and 1.4 for intracranial hemorrhage. ${ }^{4}$ As such, most studies have shown that aspirin use increases the risk of intracranial hemorrhage; however, some studies have reported that the use of aspirin has no relation to intracranial hemorrhage. ${ }^{5}$

In the chi-squared test of our study, the OR was 1.10, which showed that the risk of bleeding increased by about 10\% when using aspirin. However, in multiple logistic regression analysis, the adjusted OR was 0.81 , which was contradictory to other major previous studies, which has shown an increased risk of hemorrhage after aspirin intake. Of course, a few previous reports also reported similar results to ours with the chi-squared test. ${ }^{5,6}$ In 1996, Thrift et al. ${ }^{6}$ reported that the use of aspirin-like drugs for secondary prevention of ischemic stroke or cardiac disease was not associated with an increased risk of intracerebral hemorrhage (ICH) $(\mathrm{OR}, 0.66$; 95\% Cl, 0.20-2.21). In 2013, García Rodríguez et al. ${ }^{5}$ reported that the aspirin use was not associated with an increased risk of ICH (OR, 1.06; 95\% Cl, 0.93-1.21). However, these reports are very few, and the majority of studies differ from our findings.

The reasons why our results differ from those of most previous reports may be assumed to result from the limitations of this study. This study analyzed the HIRA-NIS 2016, an insurance claim for inpatients, and it is possible that the diagnostic code is not accurate due to the nature of the insurance claims. When defining the diagnostic code to solve this problem, it contains all the codes up to 5th in priority, but inaccuracies in the diagnostic code are still quite possible. In addition, HIRA-NIS 2016 is extracted data from patients who had been hospitalized at least once in 2016, which may also lead to bias, resulting in errors in the results. The most important disadvantage of this study is that this study analyzed data only during 2016. Although the number of subjects is sufficient, the duration of aspirin use and the follow-up period are quite limited, so errors may arise in the results. 
The results in chi-squared test were similar to those of previous studies, but multiple logistic regression analysis is usually judged to be more significant. Therefore, in conclusion, our study showed that the risk of bleeding was reduced when using aspirin. In fact, aspirin might have reduced the risk of bleeding, but this is not the same as previous reports and does not fit our experience either. These limitations are considered to be from the study mentioned above, and further studies are needed to overcome these limitations.

\section{Fund}

This work was supported by the Academic Research Fund funded by Korean Society of Interventioanl Neuroradiology.

\section{Ethics Statement}

This type of study does not require approval from the IRB.

\section{Conflicts of Interest}

The authors have no conflicts to disclose.

\section{REFERENCES}

1. Antithrombotic Trialists' (ATT) Collaboration, Baigent C, Blackwell L, Collins R, Emberson J, Godwin J, et al. Aspirin in the primary and secondary prevention of vascular disease: collaborative meta-analysis of individual participant data from randomised trials. Lancet 2009;373:1849-1860

2. Whitlock EP, Burda BU, Williams SB, Guirguis-Blake JM, Evans CV. Bleeding risks with aspirin use for primary prevention in adults: a systematic review for the U.S. Preventive services task force. Ann Intern Med 2016;164:826-835

3. De Berardis G, Lucisano G, D'Ettorre A, Pellegrini F, Lepore V, Tognoni $\mathrm{G}$, et al. Association of aspirin use with major bleeding in patients with and without diabetes. JAMA 2012;307:2286-2294

4. García Rodríguez LA, Martín-Pérez M, Hennekens CH, Rothwell PM, Lanas A. Bleeding risk with long-term low-dose aspirin: a systematic review of observational studies. PloS One 2016;11:e0160046

5. García-Rodríguez LA, Gaist D, Morton J, Cookson C, GonzálezPérez A. Antithrombotic drugs and risk of hemorrhagic stroke in the general population. Neurology 2013;81:566-574

6. Thrift AG, McNeil JJ, Forbes A, Donnan GA. Risk factors for cerebral hemorrhage in the era of well-controlled hypertension. Melbourne risk factor study (merfs) group. Stroke 1996;27:2020-2025 